\title{
Research Article \\ Erlang's Fixed-Point Approximation for Performance Analysis of HetNets
}

\author{
Guozhi Song, ${ }^{1}$ Jigang $\mathrm{Wu}^{1}{ }^{1}$ John Schormans, ${ }^{2}$ \\ and Laurie Cuthbert ${ }^{2}$ \\ ${ }^{1}$ School of Computer Science and Software Engineering, Tianjin Polytechnic University, \\ Tianjin 300387, China \\ 2 School of Electronic Engineering and Computer Science, Queen Mary, University of London, \\ London E1 4NS, UK
}

Correspondence should be addressed to Guozhi Song, guozhi.song@gmail.com

Received 17 February 2012; Accepted 16 March 2012

Academic Editor: Yonghong Yao

Copyright (C) 2012 Guozhi Song et al. This is an open access article distributed under the Creative Commons Attribution License, which permits unrestricted use, distribution, and reproduction in any medium, provided the original work is properly cited.

\begin{abstract}
We consider the analytic modelling of wireless systems with multiple access technologies in the perspective of teletraffic engineering and provide a framework for the performance analysis and evaluation of a wireless HetNet (heterogeneous network) system with both cellular and WLAN access technologies. In particular, an approach with Erlang's fixed-point approximation to calculate the new call blocking and handover call dropping probabilities in such systems is introduced. The model is versatile enough to cover not only cellular/WLAN HetNet systems but other wireless HetNets with difference access technologies in general.
\end{abstract}

\section{Introduction}

The term HetNet (heterogeneous network) used in wireless networks indicates the usage of multiple types of access technologies for a wireless system, and these radio access networks (RAN) can form a motley of macrocells, picocells, and femtocells in order to offer wireless service in an environment with a wide variety of wireless coverage zones, ranging from an open outdoor environment to office buildings, homes, and underground spaces. Therefore it has a hierarchical structure with overlapped and overlaid wireless service areas and complex interoperation between these macrocells and smaller cells (sometimes in the form of WLAN) working together to provide a mosaic of coverage. Currently the extensive research of HetNets can be basically categorized into three groups. The first group focuses on the new architecture and implementation of HetNet system such as the work Li et al. did in [1]. The second group is trying to find the good and optimized RAN selection algorithms like what 
Wang et al. did in [2] and Mapp et al. in [3]. The last group works on the system performance analysis of HetNets, for instance, the work of Xie and Baras on throughput in [4] and Wang and $\mathrm{Du}$ on routing in [5]. In this paper, we will discuss the application of Erlang's fixedpoint approximation in the performance analysis of HetNet systems which is modelled as a product-form loss network.

Ross indicated in [6] that the efficient algorithms for exact blocking probabilities and other performance measures do not exist for all product-form loss networks. The reduced fixed-point approximation is a method of approximating blocking probability in productform loss networks. And loss networks are a special kind of queueing networks (or network of queues) without any queueing (or buffer) at each node. Traditionally for a single loss system which is actually an $\mathrm{M} / \mathrm{M} / \mathrm{n} / \mathrm{n}$ queue such as a telephony system, the loss probability is estimated using Erlang's function that is discussed in Erlang's classical works [7, 8] which also laid the foundation for teletraffic engineering. It is applicable to a single-call-type system. But for our HetNet systems we adopt the loss network model with the help of reduced fixedpoint approximation that is being used in the studies of TCP behaviour [9], optical burst switched networks [10] and also routing [11], and so forth.

What is often referred to as reduced load approximation is a fairly broad class of approximation techniques. The general approach is to diminish the arrival rates of offered traffic to a subnetwork (which may be a single link) by a factor equal to the probability that a new call on that route would not be blocked on the other link of its path. Typically this method leads to a set of fixed-point equations for which exists a (not necessarily unique) solution. The underlying assumption is that of independent blocking between the individual subnetworks and, although invalid in most nontrivial situations, does yield particularly good results when traffic correlations are small.

The famous Erlang's fixed-point approximation (EFPA) is a member of the reduced load class, one which analyses each link as a separate subnetwork. The EFPA performs well asymptotically. Kelly in [12] proved that the estimates for a network with fixed routing and no controls tend towards the exact probabilities (i) when the link capacities and arrival rates are increased simultaneously keeping the network topology fixed (Kelly limiting regime), and (ii) when the number of links and routes are increased while the link loads are held constant (diverse routing limit) proved by Ziedins and Kelly in [13].

The EFPA is a solution to the set of fixed-point equations:

$$
\begin{gathered}
B_{j}=E\left(\rho_{j}, C_{j}\right), \quad j=1, \ldots, J, \\
\rho_{j}=\sum_{r \in R} a_{j, r} v_{r} \prod_{i \in r \backslash\{j\}}\left(1-B_{i}\right), \quad j=1, \ldots, J,
\end{gathered}
$$

where Erlang's formula, $E(v, C)$, gives the probability that the Erlang loss model is fully utilised and is given by

$$
E(v, C)=\frac{v^{C}}{C !}\left(\sum_{n=0}^{C} \frac{v^{n}}{n !}\right)^{-1}
$$

The interpretation is that $B_{j}$ is the probability that link $j$ is full given its offered traffic load is $\rho_{j} \cdot \rho_{j}$ is an approximation obtained by considering the carried traffic on link $j$ : the throughput 


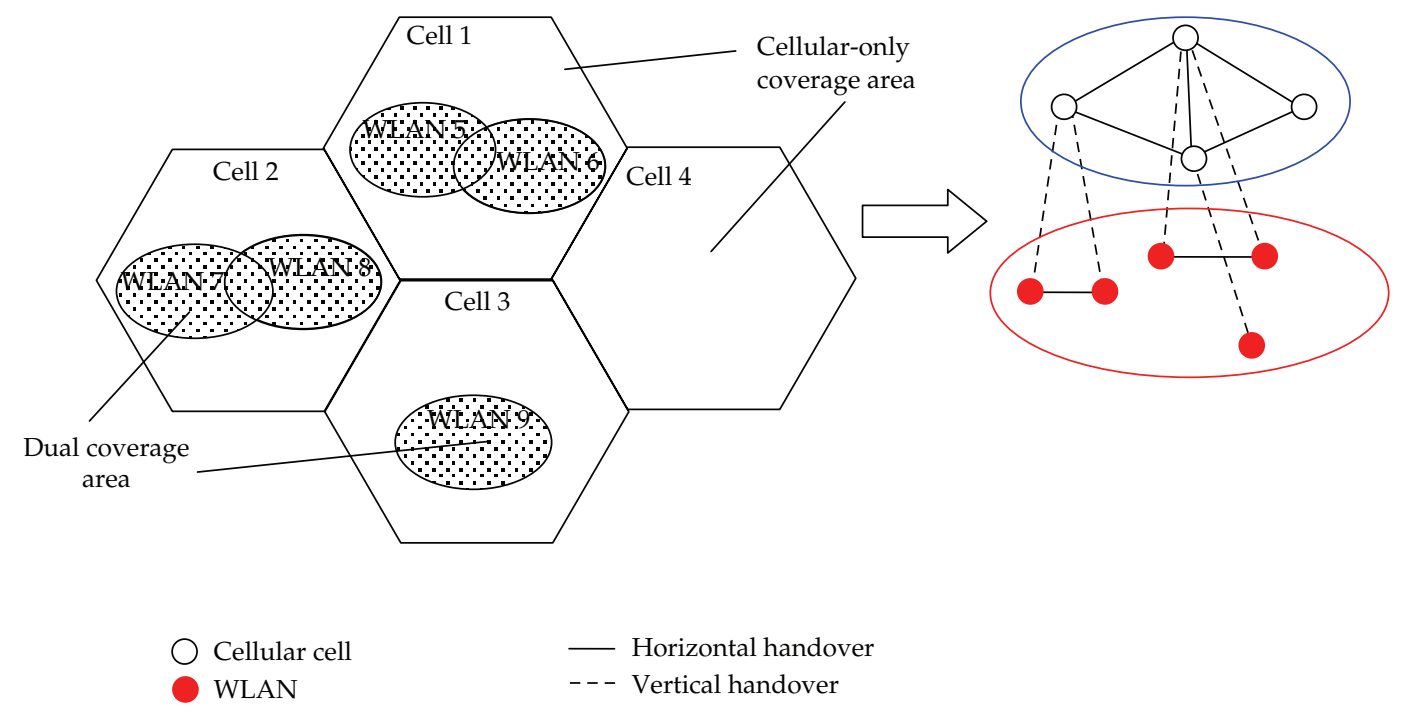

Figure 1: A HetNet (cellular/WLAN) deployment and its converted network topological representation.

of link $j$ is $\left(1-B_{j}\right) \rho_{j}$ and $\sum_{r \in R} a_{j, r} v_{r}\left(1-L_{r}\right)$ is the sum of the contributions made by each route $r$ to $j$ 's carried load. Applying the independent blocking assumption yields

$$
\begin{aligned}
L_{r} & =1-\operatorname{Pr}(\text { call accepted on each link } i \in r) \\
& =1-\prod_{i \in r} \operatorname{Pr}(\text { call accepted on link } i) \\
& =1-\prod_{i \in r}\left(1-B_{i}\right),
\end{aligned}
$$

and the expression (1.3) follows by equating the two expressions for carried traffic on $j$ and substituting for $L_{r}$. Kelly in [14] proved that there is a unique vector $\left(B_{1}, \ldots, B_{J}\right) \in[0,1]^{J}$ satisfying (1.1).

\section{System Model}

Assume there are in total $N^{c}$ cells and $N^{w}$ WLANs in our HetNet system. Let us firstly define some symbols to facilitate the further analysis. Let $C_{i}^{a}$ be the set of cells adjacent to cell $i, W_{i}^{o}$ the set of WLANs inside the coverage of cell $i, W_{k}^{a}$ the set of WLANs adjacent to WLAN $k$, and $C_{k}^{o}$ the set containing the overlaying cell of WLAN $k$ that is, a dual-coverage area as show in Figure 1. This deployment was used in [15] to evaluate and compare performance between a cellular-only network and a cellular-WLAN interworking network.

The use of reservation channels or guard channels is analogous to the use of trunk reservation in fixed-wire circuit-switched networks to give priority to fresh traffic over overflow traffic [16]. So each node can be modelled as an Erlang loss system. The service time at a node would be the channel holding time in the corresponding network the node represented. 
We use the logical topology abstraction method that was proposed and developed in $[17,18]$ to derive the network topology of the system by mapping each cellular network and WLAN as nodes and handover traffic as links.

\subsection{Node Traffic Flow Analysis}

Traffic analysis of each node is based on the conservation of traffic. Traffic flows in and out of the node will be equal. The arriving traffic can be divided into four parts: new calls, horizontal handover calls, vertical handover calls, and overflow calls. Traffic either leaves the cell or WLAN normally by completion or handover, or due to the lack of resources is blocked or dropped.

The total arrival rate at cell $i$ is the sum of the arrival rate of new calls, handover calls (including horizontal handover calls from adjacent cells and vertical handover calls from the WLANs inside the cell), and overflow calls from WLANs within the cell.

Similarly, the total arrival rate at WLAN $k$ is the sum of the arrival rate of new calls, handover calls (including horizontal handover calls from adjacent WLANs and vertical handover calls from the overlaid cellular cell), and overflow calls from the overlaid cell.

The new connection arrival processes to cell $i$ and WLAN $k$ are Poisson with rates $\lambda_{i}^{(c n)}$ and $\lambda_{k}^{(w n)}$, respectively, which are independent of other arrival processes. The channel holding time of a connection in cell $i$ (i.e., the time that a user is using resources in cell $i$ ) is an exponential distributed random variable with mean $1 / \mu_{i}^{c}$. The channel holding time in WLAN $k$ is exponentially distributed with mean $1 / \mu_{k}^{w}$. Both are independent of earlier arrival times and connection holding times.

At the end of a holding time, a connection in cell $i$ of the cellular system may terminate and leave the system with probability $T_{i}^{(c)}$ or move within the system and continue in an adjacent cell or WLAN with probability $1-T_{i}^{(c)}$. The probability that a connection continues and moves to an adjacent cell of cell $i$ or WLAN $k$ inside cell $i$ is $1-T_{i}^{(c)}=\sum_{j \in C_{i}^{a}} H_{i j}^{(c c)}+$ $\sum_{k \in W_{i}^{o}} H_{i k}^{(c w)}$, where $H_{i j}^{(c c)}$ is the probability of attempting a horizontal handover to adjacent cell $j$ and $H_{i k}^{(c w)}$ is the probability of attempting a vertical handover to WLAN $k$ inside cell $i$.

Similarly, at the end of a holding time of a connection in WLAN $k$, a call may terminate and leave the system with probability $T_{k}^{(w)}$ or move within the system and continue in an adjacent WLAN or an overlay cell of the cellular system with probability $1-T_{k}^{(w)}$. The probability that a connection continues and moves to an adjacent WLAN to WLAN $k$ or overlaying cell $i$ is $1-T_{k}^{(w)}=\sum_{l \in W_{k}^{a}} H_{k l}^{(w w)}+\sum_{i \in C_{k}^{o}} H_{k i}^{(w c)}$, where $H_{k l}^{(w w)}$ is the probability of attempting a horizontal handover to adjacent WLAN $l$, and $H_{k i}^{(w c)}$ is the probability of attempting a vertical handover to overlaying cell $i$.

Each cell $i$ of the cellular system has a capacity of $C_{i}^{c}$ units of bandwidth. While each WLAN $k$ has a capacity of $C_{k}^{w}$ units of bandwidth. I define $R_{i}^{c}$ and $R_{k}^{w}$ as the reservation parameters of cell $i$ and WLAN $k$ for handover calls, respectively. They act as the admission policy to provide handover connections priority over new connections. Two admission schemes are considered: the cut-off priority and the fractional guard channel.

Let $n_{i}$ be the number of connections in cell $i$. When cell $i$ is in any of the states $n_{i} \leq$ $C_{i}^{c}-R_{i}^{c}$, it accepts new and handover calls under both admission policies. When cell $i$ is in any 


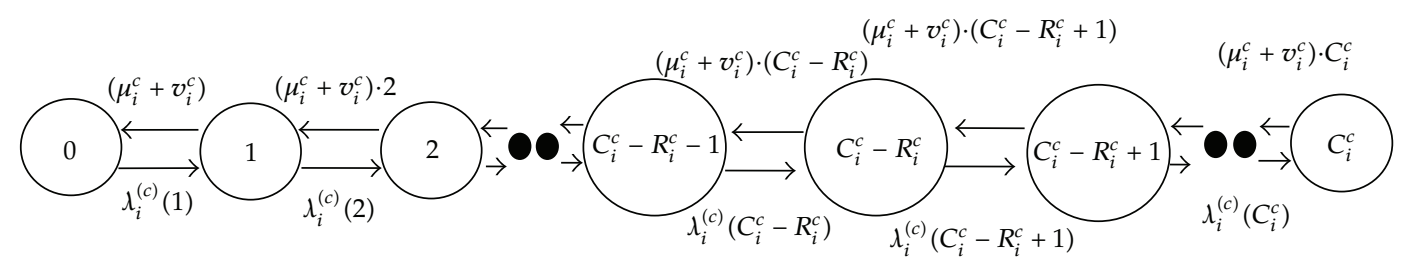

(a)

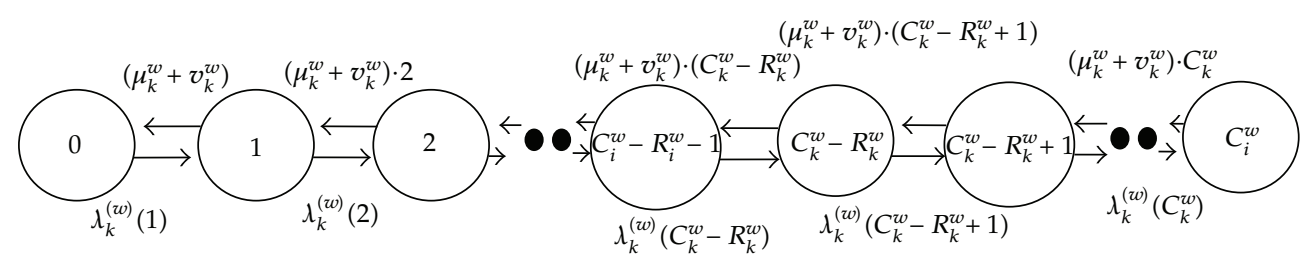

(b)

Figure 2: State transition diagrams for each cellular cell (a) and WLAN (b).

of the states $n_{i}>C_{i}^{c}-R_{i}^{c}$, only handover requests are accepted for the cut-off priority scheme. For the fractional guard channel scheme, besides handover requests, each new connection request is also accepted with probability $\omega_{i}^{c}$. The same admission policies apply to WLANs with probability $\omega_{k}^{w}$. Note that cut-off priority is a particular case of the fractional guard channel with $\omega_{k}^{w}=0$. If a handover request is not accepted in one network, then the request is transferred to the other network. In the cellular system, only when the user is inside the dualcoverage area can the connection be transferred to the WLAN. Connections that are within the WLAN can always be attempted to be transferred to the corresponding overlaying cell in the cellular system.

The occupancy of a cell evolves according to a birth-death process independent of other cells as shown in Figure 2(a). The process for cell $i$ evolves with birth rate $\rho_{i}^{c}(2.1)$ (a) for the unreserved states and $\alpha_{i}^{c}(2.1)(\mathrm{b})$ for the reserved states. The death rate of cell $i$ in state $n_{i}$ is $n_{i} \mu_{i}^{c}$. The total traffic offered to cell $i$ in state $n_{i}$ is

$$
\lambda_{i}^{c}\left(k_{i}^{c}\right)=\left\{\begin{array}{l}
\lambda_{i}^{(c n)}+\sum_{j \in C_{i}^{a}} \lambda_{j i}^{(c c)}+\sum_{k \in W_{i}^{o}} \lambda_{k i}^{(w c)}+\sum_{l \in W_{i}^{o}} \lambda_{l i}^{(w o)}, \quad k_{i}^{c} \leq C_{i}^{c}-R_{i}^{c} \quad(\mathrm{a}), \\
\lambda_{i}^{(c n)} \omega_{i}^{c}+\sum_{j \in C_{i}^{a}} \lambda_{j i}^{(c c)}+\sum_{k \in W_{i}^{o}} \lambda_{k i}^{(w c)}+\sum_{l \in W_{i}^{o}} \lambda_{l i}^{(w o)}, \quad k_{i}^{c}>C_{i}^{c}-R_{i}^{c} \quad(\mathrm{~b}),
\end{array}\right.
$$

with $\omega_{i}^{c}=0$ for the cut-off priority scheme, and $0 \leq \omega_{i}^{c} \leq 1$ for the fractional guard channel scheme. The term $\lambda_{j i}^{(c c)}$ is the horizontal handover rate of cell $j$ offered to cell $i$, for adjacent cells $i$ and $j$, and $\lambda_{k i}^{(w c)}$ is the vertical handover rate of WLAN $k$ offered to overlaid cell $i$, $\lambda_{z k}^{(c o)}$ is the proportion of all handover traffic that is not accepted in cell $z$ due to the resource depletion and thence overflowed to WLAN $k$. 


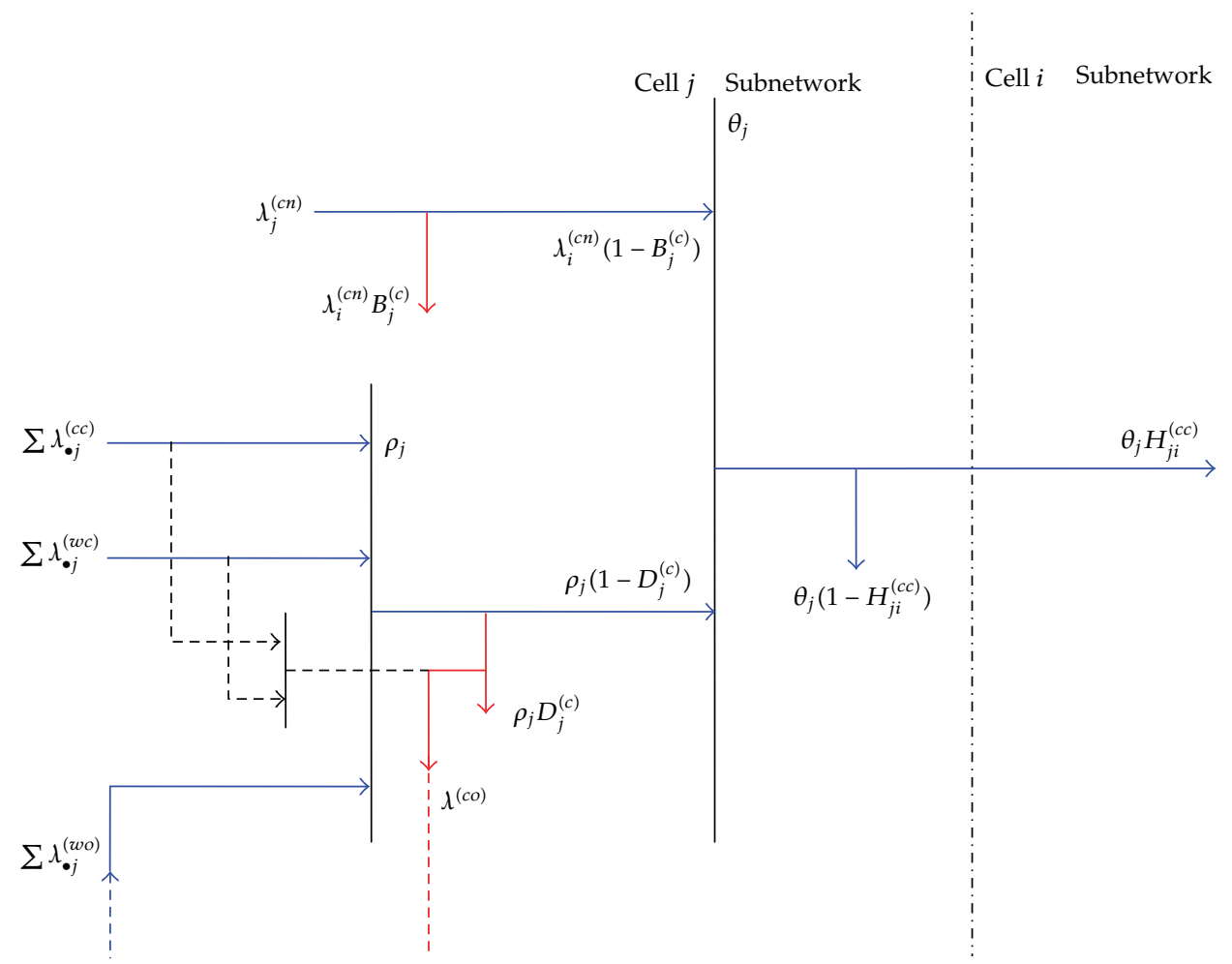

Figure 3: Horizontal handover traffic between cellular cells.

Similarly, as shown in Figure 2(b) the occupancy of WLAN $k$ evolves with birth rates $\rho_{k}^{w}(2.2)(\mathrm{a})$ and $\alpha_{k}^{w}(2.2)(\mathrm{b})$ based on the state $m_{k}$, and death rate $k_{k}^{w}\left(\mu_{k}^{w}+v_{k}^{w}\right)$. The total traffic offered to WLAN $k$ in state $k_{k}^{w}$ is

$$
\lambda_{k}^{w}\left(m_{i}^{w}\right)=\left\{\begin{array}{l}
\lambda_{k}^{(w n)}+\sum_{l \in W_{k}^{a}} \lambda_{l k}^{(w w)}+\sum_{j \in C_{k}^{o}} \lambda_{j k}^{(c w)}+\sum_{g \in C_{k}^{o}} \lambda_{g k}^{(c o)}, m_{k} \leq C_{k}^{w}-R_{k}^{w} \quad(\mathrm{a}), \\
\lambda_{k}^{(w n)} \omega_{k}^{w}+\sum_{l \in W_{k}^{a}} \lambda_{l k}^{(w w)}+\sum_{j \in C_{k}^{o}} \lambda_{j k}^{(c w)}+\sum_{g \in C_{k}^{o}} \lambda_{g k}^{(c o)}, m_{k}>C_{k}^{w}-R_{k_{i}}^{w^{c}} \quad(\mathrm{~b}),
\end{array}\right.
$$

\subsection{Link Traffic Flow (Handover Traffic) Analysis}

Let us now focus on the traffic flow on the links, that is, handover traffic and overflow traffic to derive the handover rates in (2.1) ((a), (b)) and (2.2) ((a), (b)). It will be found that the handover rates and the blocking rate and dropping rate are interdependent with each other.

\subsubsection{Handover Calls to Cell}

The horizontal handover rate $\lambda_{j i}^{(c c)}$ of cell $j$ offered to cell $i$, for adjacent cells $i$ and $j$, is all the nonblocked (dropped) traffic in cell $j$ multiply with a handover probability factor and is shown in the Figure 3. Blue lines are the traffic that can go through to the next stage, and red lines are the blocked or dropped traffic. 


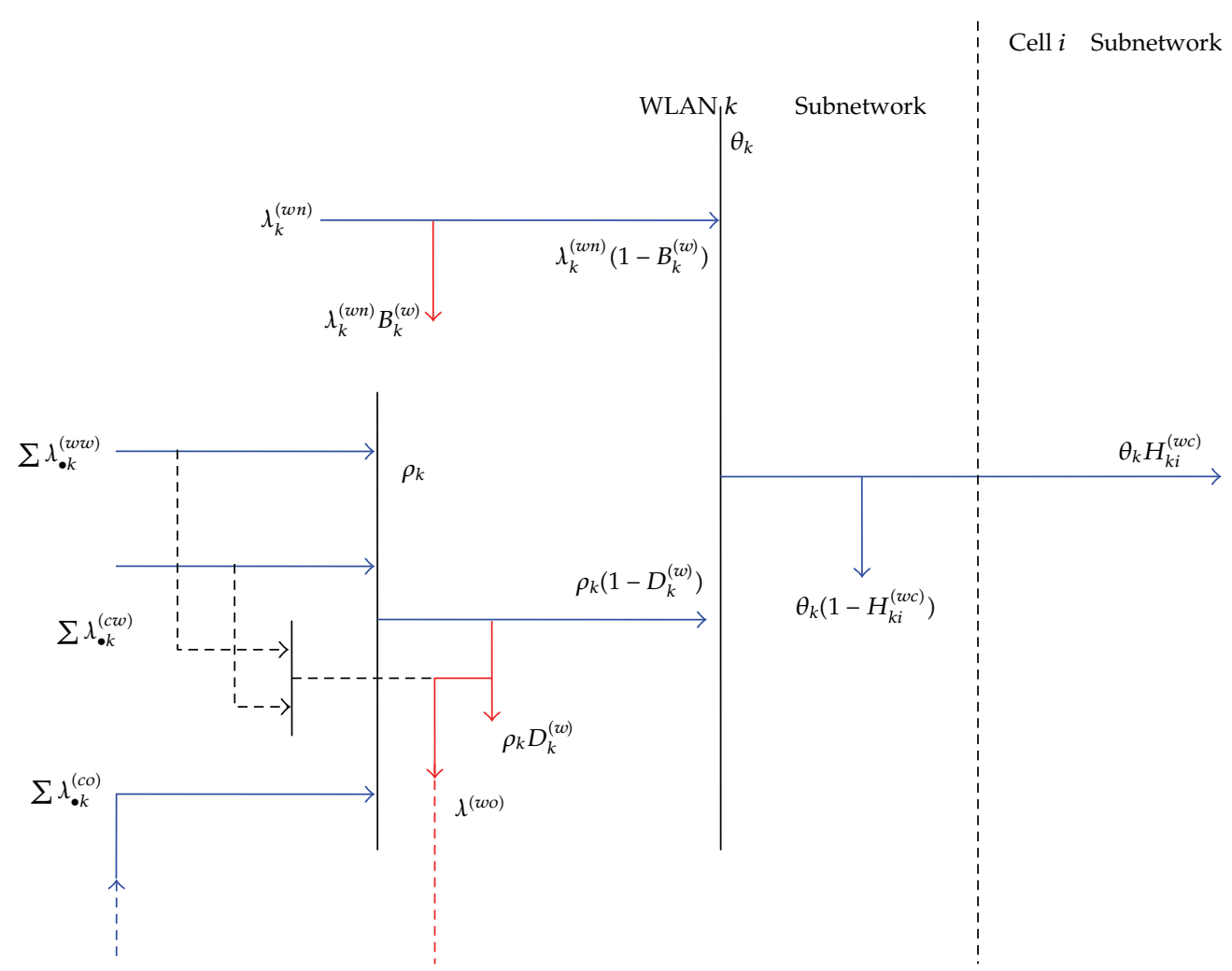

Figure 4: Vertical handover traffic from WLAN to cellular cell.

So the formula for horizontal handover is given by (2.3)

$$
\lambda_{j i}^{(c c)}=H_{j i}^{(c c)} \cdot\left[\lambda_{j}^{(c n)} \cdot\left(1-B_{j}^{(c)}\right)+\left(\sum_{x \in C_{j}^{a}} \lambda_{x j}^{(c c)}+\sum_{y \in W_{j}^{o}} \lambda_{y j}^{(w c)}+\sum_{z \in W_{j}^{o}} \lambda_{z j}^{(w o)}\right) \cdot\left(1-D_{j}^{(c)}\right)\right],
$$

where $B_{j}^{(c)}$ and $D_{j}^{(c)}$ are the new connection blocking and handover dropping probabilities in cell $j$, respectively.

In (2.3), $\lambda_{z j}^{(w o)}$ corresponds to all handover traffic that is not accepted in WLAN $z$ and hence transferred to cell $j$, so

$$
\lambda_{z j}^{(w o)}=D_{z}^{(w)} \cdot\left(\lambda_{j z}^{(c w)}+\sum_{l \in W_{z}^{a}} \lambda_{l z}^{(w w)}\right)
$$

For $\lambda_{k i}^{(w c)}$, the vertical handover rate of WLAN $k$ offered to overlay cell $i$ is all the nonblocked (dropped) traffic in WLAN $k$ multiplied with a handover probability factor as shown in the Figure 4. Blue lines are the traffic that can go through to the next stage and red 


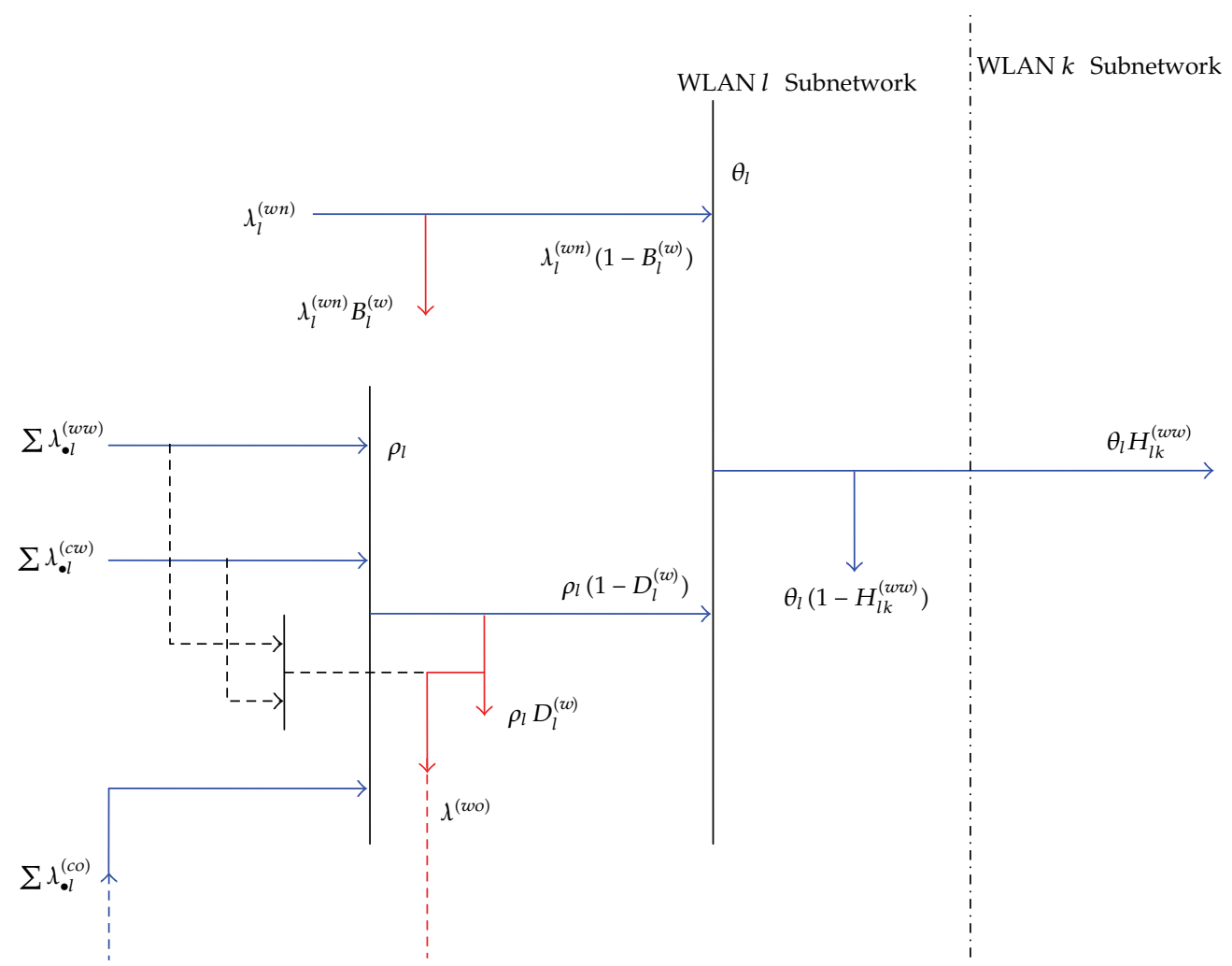

Figure 5: Horizontal handover traffic between WLANs.

lines are the blocked or dropped traffic. So the formula for vertical handover is given by (2.5)

$$
\lambda_{k i}^{(w c)}=H_{k i}^{(w c)} \cdot\left[\lambda_{k}^{(w n)} \cdot\left(1-B_{k}^{(w)}\right)+\left(\sum_{x \in W_{k}^{a}} \lambda_{x k}^{(w w)}+\sum_{y \in C_{k}^{o}} \lambda_{y k}^{(c w)}+\sum_{z \in C_{k}^{o}} \lambda_{z k}^{c o}\right) \cdot\left(1-D_{k}^{(w)}\right)\right]
$$

where $B_{k}^{(w)}$ and $D_{k}^{(w)}$ are the new connection blocking and handover dropping probabilities in WLAN $k$, respectively.

In (2.6), $\lambda_{z k}^{(c o)}$ is the proportion of all handover traffic that is not accepted in cell $z$ and thence transferred to WLAN $k$, then

$$
\lambda_{z k}^{(c o)}=D_{z}^{(c)} \cdot\left(\lambda_{k z}^{(w c)}+\sum_{l \in C_{z}^{a}} \lambda_{l z}^{(c c)}\right) \cdot \gamma_{z k}
$$

where $\gamma_{z k}$ is the coverage factor between WLAN $k$ and overlay cell $z$, that is, the ratio between the radio coverage area of WLAN $k$ and the radio coverage area of cell $z$ with $0<\gamma_{z k} \leq 1$. 


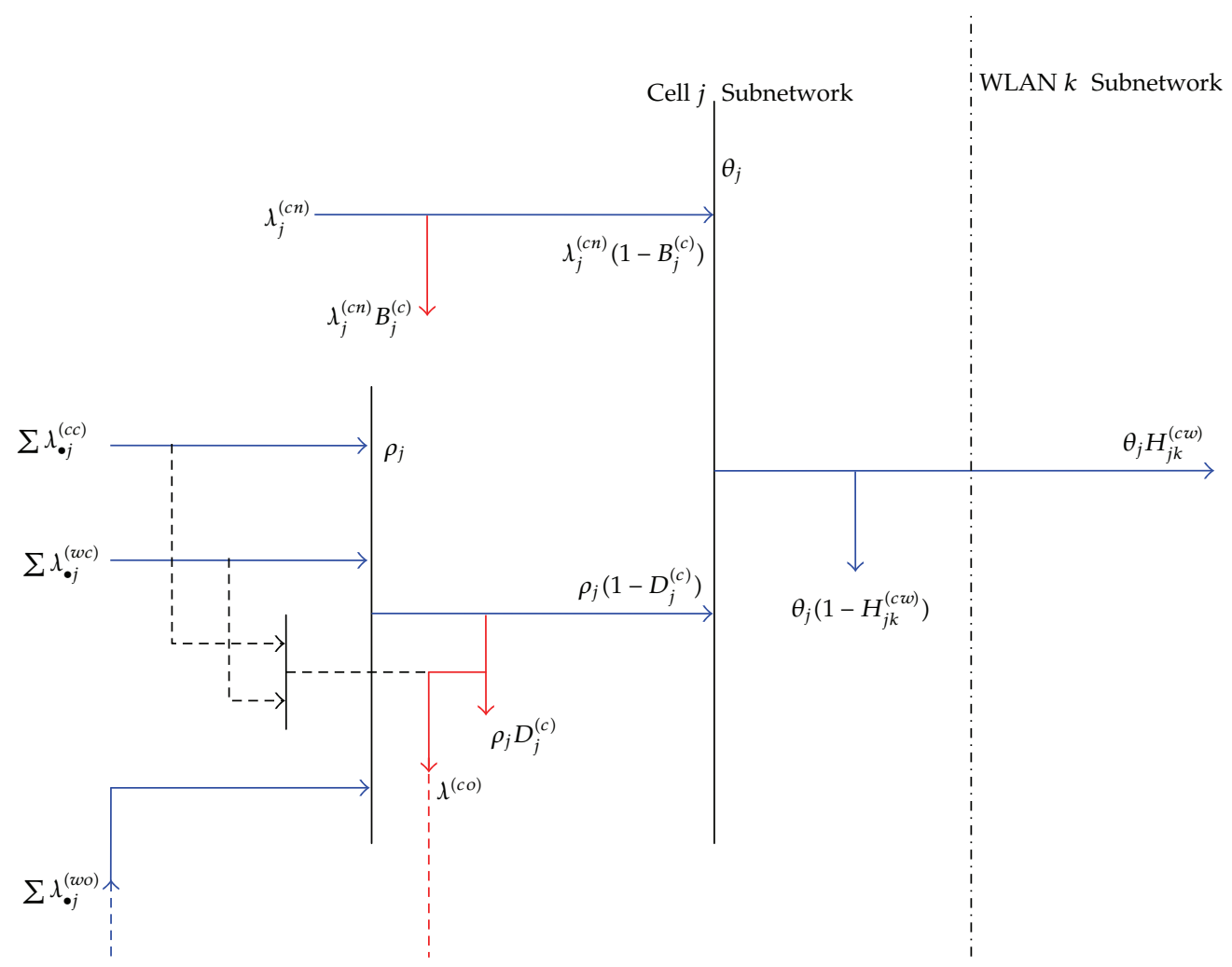

Figure 6: Vertical handover traffic from cellular cell to WLAN.

\subsubsection{Handover Calls to WLAN}

Similarly, we can derive the term $\lambda_{l k}^{(w w)}$, the horizontal handover rate of WLAN $l$ offered to WLAN $k$, for adjacent WLAN $l$ and $k$, which is all the non-blocked (dropped) traffic in WLAN multiplied with a handover probability factor as shown in the Figure 5. Blue lines are the traffic that can go through to the next stage and red lines are the blocked or dropped traffic. So the formula for horizontal handover is given by (2.7)

$$
\lambda_{l k}^{(w w)}=H_{l k}^{(w w)} \cdot\left[\lambda_{l}^{(w n)}\left(1-B_{l}^{w}\right)+\left(\sum_{x \in W_{l}^{a}} \lambda_{x l}^{(w w)}+\sum_{y \in C_{l}^{o}} \lambda_{y l}^{(c w)}+\sum_{z \in C_{l}^{o}} \lambda_{z l}^{(c o)}\right) \cdot\left(1-D_{l}^{w}\right)\right] .
$$

The term $\lambda_{j k}^{(c w)}$ is the vertical handover rate of cell $j$ offered to WLAN $k$, for overlaying cell $j$ and $k$, which is all the non-blocked (dropped) traffic in cell $j$ multiplied with a handover probability factor as shown in the Figure 6. Blue lines are the traffic that can go through to 
the next stage and red lines are the blocked or dropped traffic. So the formula for vertical handover is given by (2.8)

$$
\lambda_{j k}^{(c w)}=H_{j k}^{(c w)} \cdot\left[\lambda_{j}^{(c n)}\left(1-B_{j}^{c}\right) \gamma_{j k}+\left(\sum_{x \in C_{j}^{a}} \lambda_{x j}^{(c c)} \gamma_{j k}+\sum_{y \in W_{j}^{o}} v_{y j}^{(w c)} \gamma_{j k}+\sum_{z \in W_{j}^{o}} \lambda_{z j}^{(w o)}\right) \cdot\left(1-D_{j}^{c}\right)\right]
$$

\subsection{Balance Equations}

From the analysis of the birth-death process in cell $i$, the detailed balance equations are

$$
\begin{array}{r}
\pi_{i}^{c}\left(k_{i}^{c}-1\right) \cdot\left(\lambda_{i}^{(c n)}+\sum_{j \in C_{i}^{a}} \lambda_{j i}^{(c c)}+\sum_{k \in W_{i}^{o}} \lambda_{k i}^{(w c)}+\sum_{l \in W_{i}^{o}} \lambda_{l i}^{(w o)}\right)=\pi_{i}^{c}\left(k_{i}^{c}\right) k_{i}^{c}\left(\mu_{i}^{c}+v_{i}^{c}\right), \\
0<k_{i}^{c} \leq C_{i}^{c}-R_{i}^{c}, \\
\pi_{i}^{c}\left(k_{i}^{c}-1\right) \cdot\left(\lambda_{i}^{(c n)} \omega_{i}^{c}+\sum_{j \in C_{i}^{a}} \lambda_{j i}^{(c c)}+\sum_{k \in W_{i}^{o}} \lambda_{k i}^{(w c)}+\sum_{l \in W_{i}^{o}} \lambda_{l i}^{(w o)}\right)=\pi_{i}^{c}\left(k_{i}^{c}\right) k_{i}^{c}\left(\mu_{i}^{c}+v_{i}^{c}\right), \\
C_{i}^{c}-R<k_{i}^{c} \leq C_{i}^{c},
\end{array}
$$

where $\pi_{i}^{c}\left(k_{i}^{c}\right)$ is the steady state probability that there are $k_{i}^{c}$ calls is in cell $i$ and is derived as

$$
\begin{aligned}
\pi_{i}^{c}\left(k_{i}^{c}\right)= & \frac{\left(\lambda_{i}^{(c n)}+\sum_{j \in C_{i}^{a}} \lambda_{j i}^{(c c)}+\sum_{k \in W_{i}^{o}} \lambda_{k i}^{(w c)}+\sum_{l \in W_{i}^{o}} \lambda_{l i}^{(w o)}\right)^{k_{i}^{c}}}{\pi_{i}^{c}(0) k_{i}^{c} !\left(\mu_{i}^{c}+v_{i}^{c}\right)^{k_{i}^{c}}}, \quad n_{i} \leq C_{i}^{c}-R_{i}^{c}, \\
\pi_{i}^{c}\left(k_{i}^{c}\right)= & \frac{\left(\lambda_{i}^{(c n)}+\sum_{j \in C_{i}^{a}} \lambda_{j i}^{(c c)}+\sum_{k \in W_{i}^{o}} \lambda_{k i}^{(w c)}+\sum_{l \in W_{i}^{o}} \lambda_{l i}^{(w o)}\right)^{C_{i}^{c}-R_{i}^{c}}}{\pi_{i}^{c}(0) k_{i}^{c} !\left(\mu_{i}^{c}+v_{i}^{c}\right)^{k_{i}^{c}}}, \quad n_{i}>C_{i}^{c}-R_{i}^{c}, \\
& \times \frac{\left(\lambda_{i}^{(c n)}+\sum_{j \in C_{i}^{a}} \lambda_{j i}^{(c c)}+\sum_{k \in W_{i}^{o}} \lambda_{k i}^{(w c)}+\sum_{l \in W_{i}^{o}} \lambda_{l i}^{(w o)}\right)^{k_{i}^{c}-C_{i}^{c}+R_{i}^{c}}}{\pi_{i}^{c}(0) k_{i}^{c} !\left(\mu_{i}^{c}+v_{i}^{c}\right)^{k_{i}^{c}}}
\end{aligned}
$$


where $\pi_{i}^{c}(0)$ is the normalisation constant, obtained as:

$$
\begin{gathered}
\pi_{i}^{c}(0)=\sum_{k_{i}^{c}=0}^{C_{i}^{c}-R_{i}^{c}} \frac{1}{k_{i}^{c} !}\left(\frac{\lambda_{i}^{(c n)}+\sum_{j \in C_{i}^{a}} \lambda_{j i}^{(c c)}+\sum_{k \in W_{i}^{o}} \lambda_{k i}^{(w c)}+\sum_{l \in W_{i}^{o}} \lambda_{l i}^{(w o)}}{\mu_{i}^{c}+v_{i}^{c}}\right)^{k_{i}^{c}} \\
+\sum_{n_{i}=C_{i}^{c}-R_{i}^{c}+1}^{C_{i}^{c}} \frac{\left(\lambda_{i}^{(c n)}+\sum_{j \in C_{i}^{a}} \lambda_{j i}^{(c c)}+\sum_{k \in W_{i}^{o}} \lambda_{k i}^{(w c)}+\sum_{l \in W_{i}^{o}} \lambda_{l i}^{(w o)}\right)^{C_{i}^{c}-R_{i}^{c}}}{k_{i}^{c} !\left(\mu_{i}^{c}+v_{i}^{c}\right)^{n_{i}}} \\
\times \frac{\left(\lambda_{i}^{(c n)} \omega_{i}^{c}+\sum_{j \in C_{i}^{a}} \lambda_{j i}^{(c c)}+\sum_{k \in W_{i}^{o}} \lambda_{k i}^{(w c)}+\sum_{l \in W_{i}^{o}} \lambda_{l i}^{(w o)}\right)^{k_{i}^{c}-C_{i}^{c}+R_{i}^{c}}}{k_{i}^{c} !\left(\mu_{i}^{c}+v_{i}^{c}\right)^{n_{i}}} .
\end{gathered}
$$

From the process WLAN $k$, the detailed balance equations are

$$
\begin{array}{r}
\pi_{k}^{w}\left(k_{k}^{w}-1\right)\left(\lambda_{k}^{(w n)}+\sum_{l \in W_{k}^{a}} \lambda_{l k}^{(w w)}+\sum_{j \in C_{k}^{o}} \lambda_{j k}^{(c w)}+\sum_{g \in C_{k}^{o}} \lambda_{g k}^{(c o)}\right)=\pi_{k}^{w}\left(k_{k}^{w}\right) k_{k}^{w}\left(\mu_{k}^{w}+v_{k}^{w}\right), \\
0<k_{k}^{w} \leq C_{k}^{w}-R_{k}^{w}, \\
\pi_{k}^{w}\left(k_{k}^{w}-1\right)\left(\lambda_{k}^{(w n)} \omega_{k}^{w}+\sum_{l \in W_{k}^{a}} \lambda_{l k}^{(w w)}+\sum_{j \in C_{k}^{o}} \lambda_{j k}^{(c w)}+\sum_{g \in C_{k}^{o}} \lambda_{g k}^{(c o)}\right)=\pi_{k}^{w}\left(k_{k}^{w}\right) k_{k}^{w}\left(\mu_{k}^{w}+v_{k}^{w}\right), \\
C_{k}^{w}-R<k_{k}^{w} \leq C_{k}^{w},
\end{array}
$$

where $\pi_{k}^{w}\left(k_{k}^{w}\right)$ is the steady-state probability that $k_{k}^{w}$ calls is in WLAN $k$, and is derived as

$$
\begin{aligned}
\pi_{k}^{w}\left(k_{k}^{w}\right)= & \frac{\left(\lambda_{k}^{(w n)}+\sum_{l \in W_{k}^{a}} \lambda_{l k}^{(w w)}+\sum_{j \in C_{k}^{o}} \lambda_{j k}^{(c w)}+\sum_{g \in C_{k}^{o}} \lambda_{g k}^{(c o)}\right)^{k_{k}^{w}}}{\pi_{k}^{w}(0) k_{k}^{w} !\left(\mu_{k}^{w}+v_{k}^{w}\right)^{k_{k}^{w}}}, \quad k_{k}^{w} \leq C_{k}^{w}-R_{k}^{w}, \\
\pi_{k}^{w}\left(k_{k}^{w}\right)= & \frac{\left(\lambda_{k}^{(w n)}+\sum_{l \in W_{k}^{a}} \lambda_{l k}^{(w w)}+\sum_{j \in C_{k}^{o}} \lambda_{j k}^{(c w)}+\sum_{g \in C_{k}^{o}} \lambda_{g k}^{(c o)}\right)^{C_{k}^{w}-R_{k}^{w}}}{\pi_{k}^{w}(0) k_{k}^{w} !\left(\mu_{k}^{w}+v_{k}^{w}\right)^{k_{k}^{w}}} \\
& \times \frac{\left(\lambda_{k}^{(w n)} \omega_{k}^{w}+\sum_{l \in W_{k}^{a}} \lambda_{l k}^{(w w)}+\sum_{j \in C_{k}^{o}} \lambda_{j k}^{(c w)}+\sum_{g \in C_{k}^{o}} \lambda_{g k}^{(c o)}\right)^{k_{k}^{w}-C_{k}^{w}+R_{k}^{w}}}{\pi_{k}^{w}(0) k_{k}^{w} !\left(\mu_{k}^{w}+v_{k}^{w}\right)^{k_{k}^{w}}}, \quad k_{k}^{w}>C_{k}^{w}-R_{k}^{w},
\end{aligned}
$$


where $\pi_{k^{\prime}}^{w}(0)$ is the normalisation constant, obtained as

$$
\begin{gathered}
\pi_{k}^{w}(0)=\sum_{k_{k}^{w}=0}^{C_{k}^{w}-R_{k}^{w}} \frac{1}{k_{k}^{w} !}\left(\frac{\lambda_{k}^{(w n)}+\sum_{l \in W_{k}^{a}} \lambda_{l k}^{(w w)}+\sum_{j \in C_{k}^{o}} \lambda_{j k}^{(c w)}+\sum_{g \in C_{k}^{o}} \lambda_{g k}^{(c o)}}{\mu_{k}^{w}+v_{k}^{w}}\right)^{k_{k}^{w}} \\
+\sum_{k_{k}^{w}=C_{k}^{w}-R_{k}^{w}+1}^{C_{k}^{w}} \frac{\left(\lambda_{k}^{(w n)}+\sum_{l \in W_{k}^{a}} \lambda_{l k}^{(w w)}+\sum_{j \in C_{k}^{o}} \lambda_{j k}^{(c w)}+\sum_{g \in C_{k}^{o}} \lambda_{g k}^{(c o)}\right)^{C_{k}^{w}-R_{k}^{w}}}{k_{k}^{w} !\left(\mu_{k}^{w}+v_{k}^{w}\right)^{k_{k}^{w}}} \\
\times \frac{\left(\lambda_{k}^{(w n)} \omega_{k}^{w}+\sum_{l \in W_{k}^{a}} \lambda_{l k}^{(w w)}+\sum_{j \in C_{k}^{o}} \lambda_{j k}^{(c w)}+\sum_{g \in C_{k}^{o}} \lambda_{g k}^{(c o)}\right)^{k_{k}^{w}-C_{k}^{w}+R_{k}^{w}}}{k_{k}^{w} !\left(\mu_{k}^{w}+v_{k}^{w}\right)^{k_{k}^{w}}} .
\end{gathered}
$$

\section{QoS Metrics}

In the cellular system the new-connection blocking probability $B_{i}^{(c)}$ in cell $i$ is $B_{i}^{(c)}=$ $\sum_{k_{i}^{c}=C_{i}^{c}-R_{i}^{c}}^{C_{i}^{c}-1} \pi_{i}^{c}\left(n_{i}\right)\left(1-\omega_{i}^{c}\right)+\pi_{i}^{c}\left(C_{i}^{c}\right)$, and the handover dropping probability $D_{i}^{(c)}=\pi_{i}^{c}\left(C_{i}^{c}\right)$.

Similarly, $B_{k}^{(w)}$ in WLAN $k$ is $B_{k}^{(w)}=\sum_{K_{k}^{w}=C_{k}^{w}-R_{k}^{w}}^{C^{w}-1} \pi_{k}^{w}\left(m_{k}\right)\left(1-\omega_{k}^{w}\right)+\pi_{k}^{w}\left(C_{k}^{w}\right)$, and $D_{k}^{(w)}=$ $\pi_{k}^{w}\left(C_{k}^{w}\right)$.

As we can see, blocking probabilities $B_{i}^{(c)}, B_{k}^{(w)}$ and dropping probabilities $D_{i}^{(c)}, D_{k}^{(w)}$ and handover rates are interdependent, so they can be calculated numerically using Erlang fixed-point approximation we have discussed.

Blocking probabilities $B_{i}^{(c)}, B_{k}^{(w)}$ and dropping probabilities $D_{i}^{(c)}, D_{k}^{(w)}$ are given an initial value, then the values of the handover rates in each cellular cell and WLAN can be calculated. With handover rates we then get a new set of blocking probabilities $B_{i}^{(c)}, B_{k}^{(w)}$ and dropping probabilities $D_{i}^{(c)}, D_{k}^{(w)}$ using the Erlang-B formula. Then we compare the new blocking and dropping probabilities with the old ones; if they are less than a given threshold, they can be accepted; otherwise all the above steps will be repeated once again using the obtained blocking and dropping probabilities as the new initial inputs.

\section{Conclusions}

In this paper, we discussed the methods of reduced load approximation especially Erlang fixed-point approximation and its application in the analysis of wireless HetNet. We analysed a single-rate traffic cellular/WLAN integrated wireless network with detailed study of the node traffic flow and link traffic analysis based on a network topology scheme. QoS metrics in terms of new call blocking probability and handover call dropping probability were considered in the model and calculated using the Erlang fixed-point approximation. This transforms the network problem into finally a set of nonlinear equations. By solving this set of equations the performance of the system in terms of new call blocking probability and handover call dropping probability can be easily derived. 


\section{Acknowledgments}

This work is supported by the Tianjin Higher Education Fund for Science and Technology

Development under Grant no. 20110808, and the National Natural Science Foundation of China (NSFC) under Grants no. 60970016 and no. 61173032.

\section{References}

[1] H. Li, J. Hajipour, A. Attar, and V. C. M. Leung, "Efficient HetNet implementation using broadband wireless access with fiber-connected massively distributed antennas architecture," IEEE Wireless Communications, vol. 18, no. 3, Article ID 5876503, pp. 72-78, 2011.

[2] J. Wang, J. Liu, D. Wang, J. Pang, and G. Shen, "Optimized fairness cell selection for 3GPP LTE-A macro-pico HetNets," in Proceedings of the IEEE 74th Vehicular Technology Conference (VTC fall '11), pp. 1-5, San Francisco, Calif, USA, 2011.

[3] G. Mapp, F. Shaikh, M. Aiash, R. P. Vanni, M. Augusto, and E. Moreira, "Exploring efficient imperative handover mechanisms for heterogeneous wireless networks," in Proceedings of the 12th International Conference on Network-Based Information Systems (NBiS '09), pp. 286-291, August 2009.

[4] H. Xie and J. S. Baras, "Performance analysis of PNNI routing in ATM networks: hierarchical reduced load approximation," in Proceedings of the 1997 MILCOM Conference, vol. 2, pp. 995-1002, November 1997.

[5] R. Wang and Y. Du, "Het-Net throughput analysis with picocell interference cancellation," in Proceedings of the IEEE International Conference on Communications Workshops (ICC '11), pp. 1-6, Kyoto, Japan, June 2011.

[6] K. W. Ross, Multiservice Loss Models for Broadband Telecommunication Networks, Springer-Velrlag, Berlin, Germany, 1995.

[7] A. K. Erlang, "The theory of probabilities and telephone conversations," Nyt Tidsskrift For Matematik, vol. 20, pp. 33-39, 1909

[8] A. K. Erlang, "Solution of some problems in the theory of probabilities of significance in automatic telephone exchanges," Elektrotkeknikeren, vol. 13, pp. 5-13, 1917.

[9] T. Bu and D. Towsley, "Fixed point approximations for TCP behavior in an AQM network," in Proceedings of the Joint International Conference on Measurement and Modeling of Computer Systems (ACM SIGMETRICS '01), pp. 216-225, Cambridge, Mass, USA, June 2001.

[10] Z. Rosberg, H. L. Vu, M. Zukerman, and J. White, "Blocking probabilities of optical burst switching networks based on reduced load fixed point approximations," in Proceedings of the 22nd Annual Joint Conference on the IEEE Computer and Communications Societies (INFOCOM '03), vol. 3, pp. 2008-2018, April 2003.

[11] M. Liu and J. S. Baras, "Fixed point approximation for multirate multihop loss networks with statedependent routing," IEEE/ACM Transactions on Networking, vol. 12, no. 2, pp. 361-374, 2004.

[12] F. P. Kelly, "Loss networks," The Annals of Applied Probability, vol. 1, no. 3, pp. 319-378, 1991.

[13] I. B. Ziedins and F. P. Kelly, "Limit theorems for loss networks with diverse routing," Advances in Applied Probability, vol. 21, no. 4, pp. 804-830, 1989.

[14] F. P. Kelly, "Blocking probabilities in large circuit-switched networks," Advances in Applied Probability, vol. 18, no. 2, pp. 473-505, 1986.

[15] L. Yang, G. Song, and J. Wu, "A performance evaluation of cellular/WLAN integrated networks," in Proceedings of the 4th International Symposium on Parallel Architectures, Algorithms and Programming (PAAP '11), pp. 131-135, 2011.

[16] D. E. Everitt, "Traffic engineering of the radio interface for cellular mobile networks," Proceedings of the IEEE, vol. 82, no. 9, pp. 1371-1382, 1994.

[17] G. Song, L. Cuthbert, and J. Schormans, “Queueing network topology for modelling cellular/wireless LAN interworking systems," in Mobility Management and Quality-of-Service for Heterogeneous Networks, D. D. Kouvatsos, Ed., pp. 269-285, River Publishers, London, UK, 2009.

[18] G. Song, J. Wu, J. Schormans, L. Yang, and L. Cuthbert, "A performance study of hierarchical heterogeneous wireless integrated Networks," Journal of Applied Mathematics \& Information Sciences, vol. 6, no. 2S, pp. 497-504, 2012. 


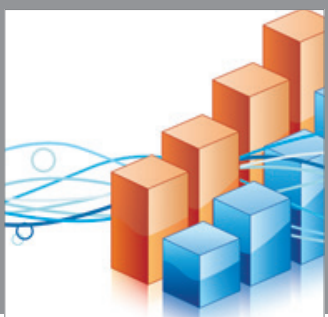

Advances in

Operations Research

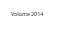

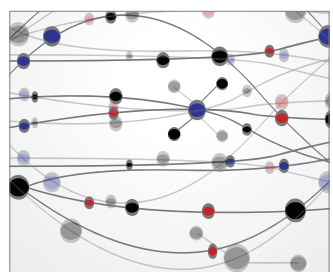

\section{The Scientific} World Journal
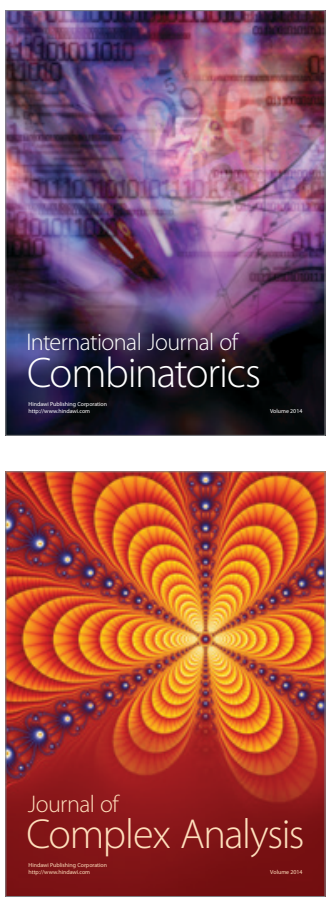

International Journal of

Mathematics and

Mathematical

Sciences
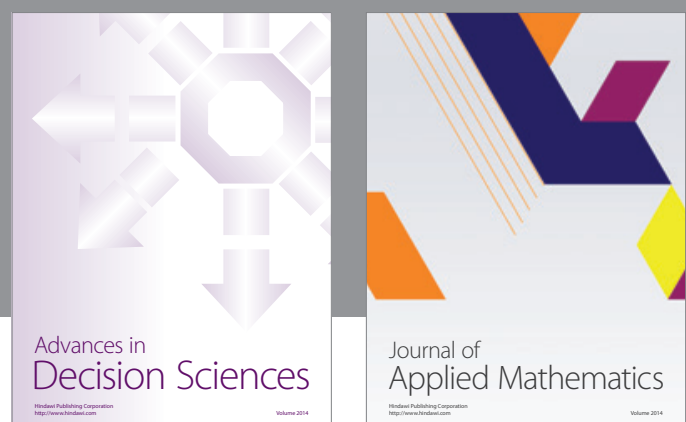

Journal of

Applied Mathematics
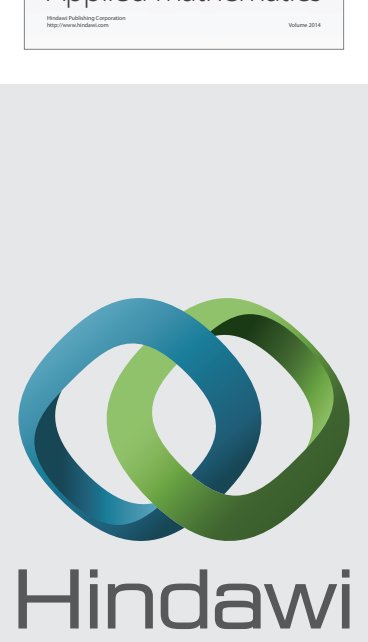

Submit your manuscripts at http://www.hindawi.com
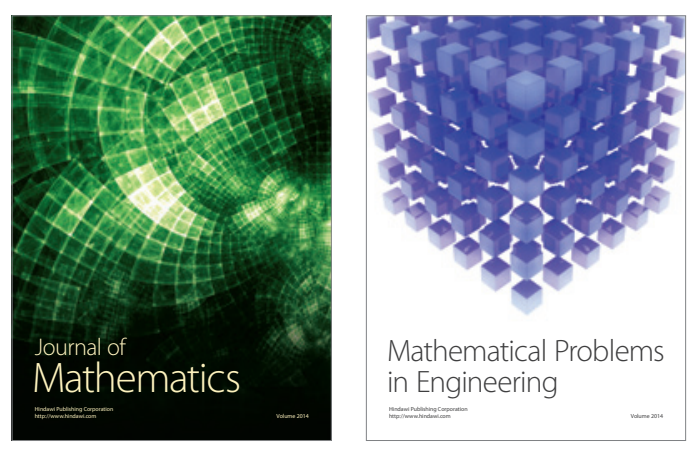

Mathematical Problems in Engineering
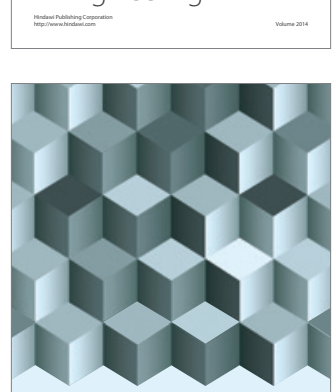

Journal of

Function Spaces
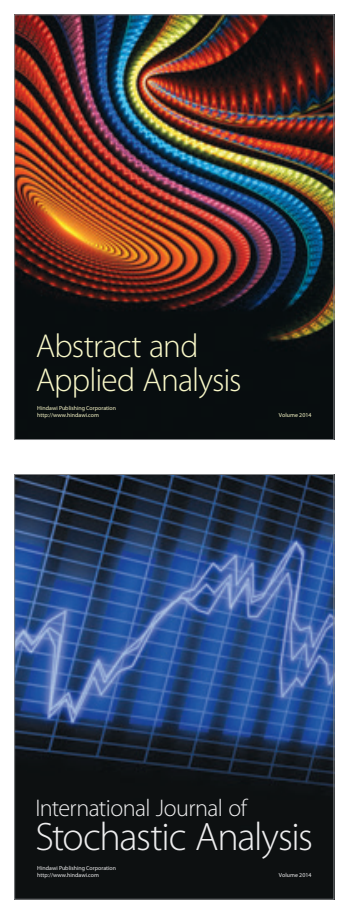

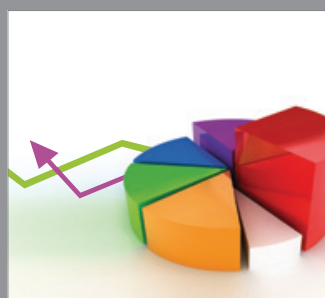

ournal of

Probability and Statistics

Promensencen
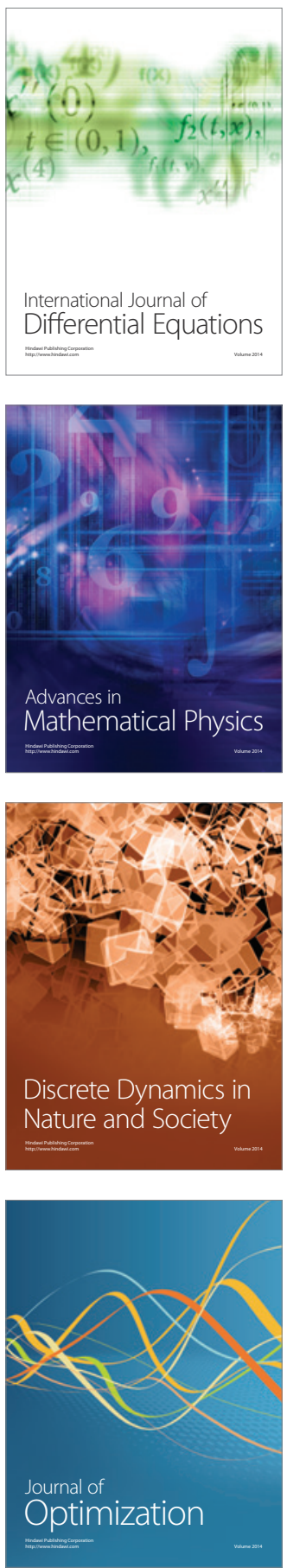